\title{
The biased odd cycle game
}

\author{
Asaf Ferber $^{1} \quad$ Roman Glebov $^{2} \quad$ Michael Krivelevich $^{13}$ \\ Hong Liu ${ }^{4} \quad$ Cory Palmer ${ }^{5} \quad$ Tomáš Valla $^{6} \quad$ Máté Vizer $^{7}$
}

Submitted: Oct 19, 2012; Accepted: Apr 8, 2013; Published: Apr 17, 2013

\begin{abstract}
In this paper we consider biased Maker-Breaker games played on the edge set of a given graph $G$. We prove that for every $\delta>0$ and large enough $n$, there exists a constant $k$ for which if $\delta(G) \geqslant \delta n$ and $\chi(G) \geqslant k$, then Maker can build an odd cycle in the $(1: b)$ game for $b=O\left(\frac{n}{\log ^{2} n}\right)$. We also consider the analogous game where Maker and Breaker claim vertices instead of edges. This is a special case of the following well known and notoriously difficult problem due to Duffus, Euczak and Rödl: is it true that for any positive constants $t$ and $b$, there exists an integer $k$ such that for every graph $G$, if $\chi(G) \geqslant k$, then Maker can build a graph which is not $t$-colorable, in the $(1: b)$ Maker-Breaker game played on the vertices of $G$ ?
\end{abstract}

Keywords: Maker-Breaker games, DLR conjecture, odd cycle game

\section{Introduction}

Let $X$ be a finite set and let $\mathcal{F} \subseteq 2^{X}$ be the family of subsets of $X$. Let $a$ and $b$ be positive integers. In an $(a: b)$ Maker-Breaker game $(X, \mathcal{F})$, two players, Maker and Breaker, take

\footnotetext{
${ }^{1}$ School of Mathematical Sciences, Raymond and Beverly Sackler Faculty of Exact Sciences, Tel Aviv University, Tel Aviv, 69978, Israel. \{ferberas,krivelev\}@post.tau.ac.il

${ }^{2}$ Institut für Mathematik, Freie Universität Berlin, Arnimallee 3-5, D-14195 Berlin, Germany, glebov@math.fu-berlin.de. Research supported by DFG within the research training group "Methods for Discrete Structures".

${ }^{3}$ Research supported in part by USA-Israel BSF Grant 2010115 and by grant 1063/08 from the Israel Science Foundation.

${ }^{4}$ Department of Mathematical Sciences, University of Illinois at Urbana-Champaign, Urbana, Illinois 61801 USA. \{hliu36, ctpalmer\}@illinois.edu

${ }^{5}$ Research supported in part by OTKA Grant NK78439.

${ }^{6}$ Czech Technical University in Prague, Faculty of Information Technology, Thákurova 9, 160 00, Prague 6, Czech Republic, tomas.valla@fit.cvut.cz. Supported by the Centre of Excellence - Inst. for Theor. Comp. Sci., Prague (project P202/12/G061 of GA ČR).

${ }^{7}$ Alfréd Rényi Institute of Mathematics, P.O.B. 127, Budapest H-1364, Hungary. vizer.mate@renyi.mta.hu
} 
turns claiming previously unclaimed elements of $X$. The game ends when every element of $X$ has been claimed by a player. Maker claims exactly $a$ board elements per turn and Breaker claims exactly $b$ board elements per turn. Unless explicitly stated otherwise, we will assume that Maker is the first player. Maker wins the game if he is able to fully claim some element of $\mathcal{F}$; otherwise Breaker wins. The set $X$ is often referred to as the board of the game and the members of $\mathcal{F}$ are referred to as the winning sets.

It is natural to play a Maker-Breaker game on the edge set of a given graph $G$. In this case the board is $X=E(G)$ and the winning sets are all the edge sets of subgraphs $H \subseteq G$ which possess a graph property $\mathcal{P}$ of special interest. For example, in the connectivity game, the winning sets are all the edge sets of subgraphs $H \subseteq G$ which contain a spanning tree of $G$. Given a positive integer $k$, in the non- $k$-colorability game, $\mathcal{N C}_{k}(G)$, the winning sets are all the edge sets of subgraphs $H \subseteq G$ which are not $k$-colorable. Since a graph $G$ is not 2-colorable if and only if it contains an odd cycle, we refer to $\mathcal{N C}_{2}(G)$ as the odd cycle game.

The following question is due to Duffus, Łuczak and Rödl [4]:

Question 1.1 Is it true that for any positive integers $k$ and $b$, there exists an integer $r=r(k, b)$ such that Maker has a winning strategy for the $(1: b)$ game $\mathcal{N C}_{k}(G)$, played on any graph $G$ such that $\chi(G) \geqslant r$ ?

Not much is known about Conjecture 1.1. By a strategy stealing argument the answer for Question 1.1 is "yes" for $b=1$ and any $k$ (for more details the reader is referred to [1]). Note that strategy stealing is a purely existential argument; we do not know any explicit strategy for Maker to win the game with these parameters.

For any $b \geqslant 2$ and any $k \geqslant 2$ Question 1.1 is open. Two partial results were obtained in [1]. The first result shows that $\chi(G)=\Omega(\log (|V(G)|))$ suffices to ensure Maker's win. The second result shows that if $\chi(G) \geqslant r$ holds in some robust way, then Maker has a winning strategy for the game on $G$. However, no bounds on $\chi(G)$ which do not depend on $|V(G)|$ are known.

In [4], Duffus, Łuczak and Rödl ask the following question:

Question 1.2 Is it true that there exists an integer $k$ such that for all graphs $G$ with $\chi(G) \geqslant k$, Maker has a strategy to claim an odd cycle in the game where Maker claims one vertex each turn and Breaker claims two?

In this paper we partially answer Questions 1.1 and 1.2 for the case where Maker's goal is to build an odd cycle (a non-2-chromatic graph), playing on a graph $G$ with high minimum degree. Our main results are the following:

Theorem 1.3 For every $0<\delta<1$, there exists an integer $n_{0}=n_{0}(\delta)$ for which the following holds. Suppose that:

1. $G$ is a graph with $|V(G)|=n \geqslant n_{0}$, and 
2. $\delta(G) \geqslant \delta n$, and

3. $\chi(G)>32 / \delta$.

Then for every $b \leqslant \frac{\delta^{2} n}{6400\left(\log _{2} n\right)^{2}}$, Maker has a winning strategy in the $(1: b)$ game $\mathcal{N C}_{2}(G)$.

The next result shows that the bound on the chromatic number of $G$ can be lowered to the optimal $\chi(G) \geqslant 3$ if $G$ has high connectivity instead of high minimum degree. In particular, $O(\log n)$-connectivity suffices in a game with a constant Breaker's bias. (This is because every $k$-chromatic graph $G$ contains a $k$-color-critical subgraph $G_{0} \subset G$, and every $k$-color-critical graph is $(k-1)$-edge-connected. Thus Theorem 1.4 can be applied to $G_{0}$.) This reproves Theorem 1.6 of [1] for the odd cycle game.

Theorem 1.4 For every positive integer $b$, there exists an integer $n_{0}=n_{0}(b)$ for which the following holds. Suppose that:

(i) $G$ is a graph with $|V(G)|=n \geqslant n_{0}$, and

(ii) $G$ is $\left(10^{4} \log _{2} n \cdot b^{2}\left(\log _{2} b\right)^{2}\right)$-edge-connected, and

(iii) $\chi(G) \geqslant 3$.

Then Maker has a winning strategy in the $\left(1:\right.$ b) game $\mathcal{N C}_{2}(G)$.

The following theorem is the "vertex version" of Theorem!1.3, where instead of claiming edges, Maker and Breaker claim vertices of the graph $G$.

Theorem 1.5 For every $0<\delta<1$ and for every positive integer $b$, there exists an integer $n_{0}=n_{0}(\delta, b)$ for which the following holds. Suppose that:

(i) $G$ is a graph with $|V(G)|=n \geqslant n_{0}$, and

(ii) $\delta(G) \geqslant \delta n$, and

(iii) $\chi(G)>2(b+1) / \delta$.

Then Maker has a winning strategy in the $(1: b)$ odd-cycle game played on $V(G)$. Furthermore, the odd cycle that Maker builds is of constant size (depending only on $\delta$ ). 


\section{$1.1 \quad$ Notation}

The notation in this paper is standard and follows that of [3]. In particular we use the following. Given a graph $G$, denote by $V(G)$ and $E(G)$ its sets of vertices and edges, respectively. For two subsets $A, B \subseteq V(G)$, let $E_{G}(A, B)$ be the set of edges in $E(G)$ with one endpoint in $A$ and one endpoint in $B$. For a vertex $v \in V(G)$ and a subset $U \subseteq V(G)$, denote by $d_{G}(v, U)$ the number of edges from $v$ to $U$. Denote by $G[U]$ the induced subgraph on $U$ and set $U^{c}=V(G) \backslash U$. We also denote $N_{G}(U)=\{v \in V(G): \exists u \in U . v u \in E(G)\}$.

The remainder of the paper is organized as follows. In Section 2, we will list some tools to be used in our arguments. Theorems 1.3 and 1.4 are proved in Section 3. Theorem 1.5 is proved in Section 4.

\section{Preliminaries}

The following lemma shows that if $G$ is a graph with high minimum degree and high chromatic number, then it contains a subgraph which is highly connected and is only one edge far from being bipartite. This is a key ingredient in the proof of Theorem 1.3.

Lemma 2.1 Let $0<\delta<1$ and let $n$ be a sufficiently large integer. Suppose that $G$ is a graph on $n$ vertices with $\delta(G) \geqslant \delta n$ and $\chi(G)>32 / \delta$. Then there exist two disjoint subsets $A, B \subseteq V(G)$ such that the bipartite graph $H=\left(A \cup B, E_{G}(A, B)\right)$ is $\delta^{2} n / 64$-connected and $E_{G}(A) \neq \emptyset$.

To prove Lemma 2.1 we use the following lemma due to Bohman, Frieze, Krivelevich and Martin [2] which enables us to partition a dense graph into a few vertex disjoint subgraphs which are highly connected:

Lemma 2.2 (Lemma 1 in [2]) Let $H$ be a graph on $n$ vertices with minimum degree $k>0$. Then there exists a partition $V(H)=V_{1} \cup \ldots \cup V_{t}$ such that for every $1 \leqslant i \leqslant t$ the set $V_{i}$ has at least $k / 8$ vertices and the induced subgraph $H\left[V_{i}\right]$ is $k^{2} /(16 n)$-vertex-connected.

For the convenience of the reader we include the proof of Lemma 2.2.

Proof. Recall a classical result of Mader (see [3]) stating that every graph of average degree at least $k$ has a $k / 4$-vertex-connected subgraph.

Let $\left(C_{1}, \ldots, C_{t}\right)$ be a family of disjoint subsets of $V(H)$ with the property that each induced subgraph $H\left[C_{i}\right]$ is $k / 8$-connected and that, among all such families of subsets, the set of vertices

$$
C:=\bigcup_{i=1}^{t} C_{i}
$$

is maximal. According to Mader's Theorem, $t>0$. Also, $\left|C_{i}\right| \geqslant k / 8$ for all $i$ and thus $t \leqslant 8 n / k$. 
Let now $\left(V_{1}, \ldots, V_{t}\right)$ be a family of disjoint subsets of $V(H)$ such that $C_{i} \subseteq V_{i}$, the induced subgraph $H\left[V_{i}\right]$ is $k^{2} /(16 n)$-connected for all $1 \leqslant i \leqslant t$ and that among all such families the set of vertices

$$
U:=\bigcup_{i=1}^{t} V_{i}
$$

is maximal. We claim that $U=V(H)$. Assume to the contrary that there exists a vertex $v \in U^{c}$. If $\left|N_{H}(v) \cap V_{i}\right| \geqslant k^{2} /(16 n)$ for some $i$, then adding $v$ to $V_{i}$ can be easily seen to keep $H\left[V_{i}\right] k^{2} /(16 n)$-connected, contradicting the maximality of $U$. Thus $v$ has less than $k^{2} /(16 n)$ neighbors in each of the $t \leqslant 8 n / k$ sets $V_{i}$, and therefore $d\left(v, U^{c}\right)>k-$ $(8 n / k)\left(k^{2} /(16 n)\right)=k / 2$. We conclude that the minimum degree of the induced subgraph $H\left[U^{c}\right]$ is at least $k / 2$. Applying Mader's Theorem, this time to $H\left[U^{c}\right]$, unveils a $k / 8$ connected subgraph disjoint from $C$, a contradiction of the choice of $\left(C_{1}, \ldots, C_{t}\right)$. Hence the family $\left(V_{1}, \ldots, V_{t}\right)$ indeed covers all the vertices of $H$ and thus forms a required partition.

Proof of Lemma 2.1. Let $V(G)=X_{1} \cup X_{2}$ be a partition such that for every $X_{i} \in\left\{X_{1}, X_{2}\right\}$ and for every $v \in X_{i}, d\left(v, X_{3-i}\right) \geqslant d(v) / 2$ (easily obtained by taking a partition which maximizes $\left.\left|E\left(X_{1}, X_{2}\right)\right|\right)$. Consider the bipartite subgraph $G^{\prime} \subseteq G$ obtained by removing all the edges inside $X_{1}$ and $X_{2}$; clearly $\delta\left(G^{\prime}\right) \geqslant \delta(G) / 2 \geqslant \delta n / 2$. Now, applying Lemma 2.2 to $G^{\prime}$, we get a partition $V\left(G^{\prime}\right)=V_{1} \cup \ldots \cup V_{t}$ with

$$
\left|V_{i}\right| \geqslant\left(\frac{\delta n}{2}\right) / 8=\frac{\delta n}{16}
$$

hence

$$
t \leqslant \frac{n}{\delta n / 16}=\frac{16}{\delta},
$$

and the induced subgraph $G^{\prime}\left[V_{i}\right]$ is $(\delta n / 2)^{2} /(16 n)=\delta^{2} n / 64$-connected, for every $1 \leqslant i \leqslant t$. Since $\chi(G)>32 / \delta \geqslant 2 t$, we conclude that there exists $1 \leqslant i \leqslant t$ for which $G\left[V_{i}\right]$ is not 2-colorable, since otherwise giving distinct sets of 2 colors to each $G\left[V_{i}\right]$ yields a proper $2 t$-coloring, a contradiction. Recall that $G^{\prime}\left[V_{i}\right]$ is bipartite, so we can denote its parts by $A$ and $B$ in such a way that $E_{G}(A) \neq \emptyset$. This completes the proof.

The following theorem of Alon, Hefetz and Krivelevich [1] enables Maker to win the $(1: b)$ connectivity game played on the edge set of some given graph $G$, provided that $G$ is a highly edge-connected graph. It will be useful in the proof of Theorems 1.3 and 1.4.

Theorem 2.3 Let $G=(V, E)$ be a graph on $n$ vertices and let $b \geqslant 2$ and $k=k(n) \geqslant \log _{2} n$ be integers. If $G$ is $\left(100 \mathrm{~kb} \log _{2} b\right)$-edge-connected, then in the $(1: b)$ game played on $E(G)$, Maker (as a first or second player) has a strategy for building a $k$-edge-connected spanning subgraph of $G$. In particular, Maker can build a connected spanning subgraph of $G$.

For the proof of Theorem 1.5 we need the following lemmas: 
Lemma 2.4 Let $0<\delta<1, b$ be a positive integer and $n$ be a sufficiently large integer. Suppose that $G$ is a graph on $n$ vertices with $\delta(G) \geqslant \delta n$ and $\chi(G)>2(b+1) / \delta$. Then there exist two disjoint subsets $A, B \subseteq V(G)$ such that the bipartite graph $H=\left(A \cup B, E_{G}(A, B)\right)$ satisfies

(i) $\left|E_{H}\left(U, U^{c}\right)\right|=\Omega\left(n^{3 / 2}\right)$ for every $U \subseteq A \cup B$ of size $\delta n / 2 \leqslant|U| \leqslant|A \cup B|-\delta n / 2$;

(ii) $\delta(H) \geqslant \delta^{2} n / 2$;

(iii) all but $o(n)$ vertices in $H$ have degree at least $(1-o(1)) \delta n / 2$; and

(iv) $\chi(G[A])>b+1$.

In a similar way as the proof of Lemma 2.1, Lemma 2.4 is an immediate consequence of the following:

Lemma 2.5 Let $0<\delta<1$ and let $n$ be a sufficiently large integer. Let $G$ be a graph on $n$ vertices with $\delta(G) \geqslant \delta n$. Then there exists a partition $V(G)=V_{1} \cup \ldots \cup V_{t}$ which satisfies:

(i) $\left|V_{i}\right| \geqslant \delta n(1-o(1))$ for every $1 \leqslant i \leqslant t ;$

(ii) $\delta\left(G\left[V_{i}\right]\right) \geqslant \delta^{2} n$ for every $1 \leqslant i \leqslant t ;$

(iii) all but $o(n)$ vertices in $G\left[V_{i}\right]$ have degree at least $\delta n(1-o(1))$ for every $1 \leqslant i \leqslant t$; and

(iv) $\left|E_{G}(A, B)\right|=\Omega\left(n^{3 / 2}\right)$ for every partition $V_{i}=A \cup B$ for which $\delta n \leqslant|A| \leqslant\left|V_{i}\right|-\delta n$ and for every $1 \leqslant i \leqslant t$.

Proof. We construct the partition in the following way: initially, let $V_{1}:=V(G)$ and $U:=\emptyset$. Assume we have a partition $V(G)=V_{1} \cup \cdots \cup V_{k}$ and a current set $U$ (notice that $U$ will be modified during the iterations). If there exists an index $1 \leqslant i \leqslant k$ and a partition $V_{i}=A \cup B$ for which $|A|,|B| \geqslant \delta n$ and $e(A, B)<n^{3 / 2}$, then we break $V_{i}$ into $A \cup B$ and define a new partition $V_{1} \cup \cdots \cup V_{i-1} \cup A \cup B \cup V_{i+1} \cup \cdots V_{k}$. Notice that there are at most $n^{3 / 4}$ vertices $v$ in $X \in\{A, B\}$ such that $d(v, X)<d\left(v, V_{i}\right)-n^{3 / 4}$. Otherwise, we get that $e(A, B) \geqslant n^{3 / 2}$, a contradiction. We add these at most $2 n^{3 / 4}$ vertices to $U$. Since none of the $V_{i}$ 's is ever smaller than $\delta n$, we get that this procedure stops after at most $1 / \delta$ iterations. Let $V(G)=V_{1} \cup \ldots \cup V_{t}$ be the final partition and let $U$ be the corresponding set of vertices with (possibly) small degrees inside their parts. Notice that for every $i \in[t]$ and every $v \in V_{i} \backslash U$, we have $d\left(v, V_{i}\right) \geqslant \delta n-t n^{3 / 4}=\delta n(1-o(1))$. By the previous argument, in every iteration we increase $|U|$ by at most $2 n^{3 / 4}$, hence $|U| \leqslant 2 n^{3 / 4} / \delta$. Now for every vertex $v \in U$, choose a part $V_{j}, 1 \leqslant j \leqslant t$, for which $d\left(v, V_{j}\right) \geqslant \delta^{2} n$ and move $v$ to $V_{j}$ (recall that $d_{G}(v) \geqslant \delta n$ and that $t \leqslant 1 / \delta$ ). We get a final partition which satisfies properties $(i)-(i v)$.

For the next lemma, we need to define an auxiliary game. Given a graph $H$ and a subset $M \subseteq V(H)$, consider the Maker-Breaker game $\mathcal{G}(H, M)$ played on $V(H)$, in which the winning sets are all subsets $T \subseteq V(H)$, such that $H[T \cup M]$ is a connected subgraph of $H$ or it contains a triangle. 
Lemma 2.6 Let $H=\left(A \cup B, E_{G}(A, B)\right)$ be a graph satisfying $(i)$ - (iii) of Lemma 2.4. Let $M \subseteq V(H)$ be any subset that can be partitioned to $M=D \cup R$, where $D$ is any dominating set of size $O(\log n)$, and such that for each vertex $v \in D$ there exists $u \in R$ with degree at least $(1-o(1)) \delta n / 2$ such that uv $\in E(H)$. Then, for any constant $b$, Maker can win the $(1: b)$ game $\mathcal{G}(H, M)$ within $O(\log n)$ moves.

Proof. First, notice that since $|D|=O(\log n)$ we conclude that there are at most $O(\log n)$ components in $H[M]$. Now, in order to complete the proof, it suffices to prove that Maker can merge two components of $H[M]$ within two rounds, by claiming extra vertices.

Let $C$ be a component of $H[M]$. Recall that $C$ spans an edge $x y$, where $x \in D$ with $d(x) \geqslant \delta(H) \geqslant \delta^{2} n / 2$ and $y \in N_{H}(x) \backslash D$ with $d(y) \geqslant(1-o(1)) \delta n / 2$. Let $U=C \cup N_{H}(C)$. We may assume that $\left|N_{H}(x) \cup N_{H}(y)\right| \geqslant \delta n / 2$, since otherwise $\left|N_{H}(x) \cap N_{H}(y)\right|=d(x)+$ $d(y)-\left|N_{H}(x) \cup N_{H}(y)\right| \geqslant(1-o(1)) \delta^{2} n / 2$, and Maker can claim a vertex in $N_{H}(x) \cap N_{H}(y)$ to complete a triangle. Hence $|U| \geqslant\left|N_{H}(x) \cup N_{H}(y)\right| \geqslant \delta n / 2$. We consider two cases depending on the size of $U^{c}$.

Case 1: $\left|U^{c}\right| \geqslant \delta n / 2$. Then by Lemma 2.4 (i), $\left|E_{H}\left(U, U^{c}\right)\right|=\Omega\left(n^{3 / 2}\right)$. Note that edges between $U$ and $U^{c}$ can only go from $N_{H}(C) \backslash C$ to $U^{c}$. Therefore, since $\left|N_{H}(C) \backslash C\right| \leqslant n$, there exist $\Omega(\sqrt{n})$ many vertices $z \in N(C) \backslash C$ with $d\left(z, U^{c}\right)=\Omega(\sqrt{n})$. Since Breaker's bias $b$ is a constant, Maker can claim such a $z$ and one of its neighbors in $U^{c}$, say $z^{\prime}$, in two rounds. Since $z^{\prime} \in U^{c}, z^{\prime}$ is not adjacent to any vertex in $C$. However, $D \subset M$ is a dominating set of $H$, thus $z^{\prime}$ is adjacent to some vertex in $M$ contained in a component of $H[M]$ other than $C$. Thus after claiming $z$ and $z^{\prime}$, Maker merges two components of $H[M]$.

Case 2: $\left|U^{c}\right|<\delta n / 2$. We are done if $U^{c}$ is empty, thus $U^{c}$ is not empty and contains some component of $H[M]$, call it $C^{\prime}$. Similarly, $C^{\prime}$ contains two adjacent vertices $x^{\prime}, y^{\prime}$ such that $d\left(x^{\prime}\right) \geqslant \delta^{2} n / 2$ and $d\left(y^{\prime}\right) \geqslant(1-o(1)) \delta n / 2$. We may assume that $\mid\left(N(C) \cap N\left(C^{\prime}\right)\right) \backslash$ $\left(C \cup C^{\prime}\right) \mid=o(n)$, since otherwise Maker can merge two components in just one move by claiming a vertex in $N(C) \cap N\left(C^{\prime}\right)$. Thus all but $o(n)$ vertices in $N\left(x^{\prime}\right) \cup N\left(y^{\prime}\right)$ are in $U^{c}$. But $\left|U^{c}\right|<\delta n / 2$, thus $\left|N\left(x^{\prime}\right) \cap N\left(y^{\prime}\right)\right| \geqslant(1-o(1)) \delta^{2} n / 2$. Then Maker can easily claim a vertex in $N_{H}(x) \cap N_{H}(y)$ to complete a triangle.

\section{Proofs of Theorems 1.3 and 1.4}

In this section we prove Theorems 1.3 and 1.4.

Proof of Theorem 1.3. First we describe a strategy for Maker and then prove it is indeed a winning strategy. At any point during the game, if Maker cannot follow the proposed strategy, then he forfeits the game. Maker's strategy is divided into the following two stages:

Stage I: Let $H=\left(A \cup B, E_{G}(A, B)\right)$ be a subgraph of $G$ as described in Lemma 2.1 and let $e \in E_{G}(A)$. In his first move, Maker claims the edge $e$.

Stage II: In this stage, Maker builds a connected spanning subgraph of $H$. 
Notice that if Maker can follow the proposed strategy then he wins the game. Indeed, if Maker has built a connected spanning subgraph of $H$, then since $H$ is a bipartite graph and $e$ is in one of its sides then adding $e$ to Maker's graph creates an odd cycle. It thus suffices to prove that indeed Maker can follow the proposed strategy without forfeiting the game. We consider each stage separately.

Stage I: Follows immediately from Lemma 2.1 and since Maker is the first player to claim an edge.

Stage II: Apply Theorem 2.3 on $H$ with $b \leqslant \frac{\delta^{2} n}{6400\left(\log _{2} n\right)^{2}}$ and $k=\log _{2} n$, then

$$
100 k \cdot b \cdot \log _{2} b \leqslant 100 \log _{2} n \cdot \frac{\delta^{2} n}{6400\left(\log _{2} n\right)^{2}} \cdot \log _{2} n \leqslant \frac{\delta^{2} n}{64} .
$$

Thus $\mathrm{H}$ is $\left(100 k b \log _{2} b\right)$-edge-connected, and Maker has a winning strategy for the $(1: b)$ $k$-edge-connectivity game played on $E(H)$. In particular, Maker can build a connected spanning subgraph of $H$.

The idea for Theorem 1.4 is similar, we provide only a sketch here.

Proof of Theorem 1.4: Let $G$ be a graph which satisfies the conditions of Theorem 1.4. Set $k:=100 \log _{2} n \cdot b \log _{2} b$, then $G$ is $\left(100 k b \log _{2} b\right)$-edge-connected. Now we consider two cases:

Case 1: Suppose that there exists a spanning subgraph $G^{\prime} \subseteq G$ that is bipartite and $k$-edge-connected. Then since $\chi(G) \geqslant 3$, at least one side of $G^{\prime}$ spans an edge in $G$. Maker, in his first move, claims such an edge. Starting from his second move, Maker plays a connectivity game on $E\left(G^{\prime}\right)$. Let $k^{\prime}=\log _{2} n$, then $G^{\prime}$ is $k=\left(100 k^{\prime} b \log _{2} b\right)$-edge-connected. Thus by Theorem 2.3, Maker can build a $k^{\prime}$-edge-connected spanning subgraph $G^{\prime \prime} \subseteq G^{\prime}$. Then $G^{\prime \prime}$ together with the first edge Maker claimed contains an odd cycle.

Case 2: Suppose that all spanning subgraphs of $G$ that are $k$-edge-connected are nonbipartite. Then Maker plays the $k$-connectivity game on $E(G)$. By Theorem 2.3, he can build a $k$-edge-connected spanning subgraph of $G$, which is, under the assumption of Case 2, non-bipartite as desired.

\section{Proof of Theorem 1.5}

In this section we prove Theorem 1.5.

Proof. First we describe a strategy for Maker and then prove it is indeed a winning strategy. At any point during the game, if Maker cannot follow the proposed strategy, then he forfeits the game. Maker's strategy is divided into the following four stages:

Stage I: Let $H=\left(A \cup B, E_{G}(A, B)\right)$ be a subgraph of $G$ as described in Lemma 2.4. In his first two moves, Maker claims two adjacent vertices, $u$ and $v$, in $A$.

Stage II: In this stage, Maker claims a dominating set $D$ of $H$ of size $100 \log n / \delta^{2}$.

Stage III: Let $D^{\prime}=D \cup\{u, v\}$. In this stage, for every vertex $w \in D^{\prime}$, Maker claims a distinct vertex $z \in N(w) \backslash D^{\prime}$ with $d_{H}(z)=(1-o(1)) \delta n / 2$. 
Stage IV: Let $M$ be the set of vertices that Maker has claimed so far. In this stage Maker claims a set of vertices $T \subseteq V(H)$ of size $O(\log n)$, for which $H[M \cup T]$ is connected. Notice that if Maker can follow the proposed strategy then he has claimed an odd cycle. Indeed, if Maker connects $M$ in $H$, in particular Maker has built a $(u, v)$-path, say $P$, in $H$. Then since $H$ is a bipartite graph and $u$ and $v$ are in the same partition class, $P$ is of even length. Thus $P$, together with the edge $u v$ that he claimed in Stage I, form an odd cycle claimed by Maker. Furthermore, we will show that Maker can also ensure an odd cycle of constant length.

We prove that indeed Maker can follow the proposed strategy without forfeiting the game.

Stage I: By Lemma 2.4 (iv) we have $b+1<\chi(G[A]) \leqslant \Delta(G[A])+1$. Hence $\Delta(G[A]) \geqslant$ $b+1$, namely there is a star with $b+1$ leaves in $G[A]$. Since Breaker can only claim $b$ vertices at each round, Maker can claim the center of this star in his first move and then claim one of its leaves in his second move.

Stage II: We show that by claiming vertices uniformly at random, after claiming $100 \log n / \delta^{2}$ vertices, with high probability (that is, with probability $1-o(1)$ ), Maker claims a dominating set $D$ of $H$ against any strategy of Breaker. Since the game of claiming a dominating set of $H$ in $100 \log n / \delta^{2}$ moves is a finite, perfect information game (and therefore - deterministic), it follows that Maker has a deterministic strategy for claiming such a $D$ (although we do not describe it here). It suffices to show that a set $D$ of $100 \log n / \delta^{2}$ vertices chosen uniformly at random from $V(H)$ is with high probability a dominating set of $H$. Fix a vertex $x \in V(H)$, since $\delta(H) \geqslant \delta^{2} n / 2$, the probability that no vertex from $N_{H}(x)$ is chosen is at most $\left(1-\delta^{2} / 2\right)^{100 \log n / \delta^{2}}$. Applying the union bound we get

$\mathbb{P}[D$ is not a dominating set of $H] \leqslant n \cdot\left(1-\delta^{2} / 2\right)^{100 \log n / \delta^{2}} \leqslant n \cdot e^{-\frac{\delta^{2}}{2} \cdot 100 \log n / \delta^{2}}=o(1)$.

It could happen that some vertex that Maker wants to claim, when building $D$, is already taken by Breaker. However, Stages I and II take only $|D|+2=O(\log n)$ rounds. Therefore, during the first two stages Breaker claims $O(\log n)=o(n / \log n)$ many vertices. It thus follows that, in each move, the probability that Maker will choose a vertex which has already been claimed by Breaker is at most $o(1 / \log n)$. Hence, with probability $1-o(1)$ Maker never chooses any vertex that Breaker has already claimed.

Stage III: By Lemma 2.4 (ii) and (iii), every $w \in D^{\prime}$ has $\Theta(n)$ many neighbors, all but $o(n)$ of which have degree $(1-o(1)) \delta n / 2$. Since $\left|D^{\prime}\right|=O(\log n)=o(n)$, Maker can secure such a distinct neighbor for each vertex in $D^{\prime}$.

Stage IV: Let $F$ be the set of vertices that Breaker has claimed in Stage I, II and III. Notice that $|F|=O(\log n)=o(n)$ has negligible size. Applying Lemma 2.6 to $H \backslash F$ gives us the desired result.

We will finish the proof by showing that the odd cycle that Maker built is of constant length. Observe that with high probability $D$ from Stage II has the following property:

(P1) $\forall v \in V(H), d(v, D) \geqslant 25 \log _{2} n$.

Consider a vertex $v$ in $H$. Since $\delta(H) \geqslant \delta^{2} n / 2$, for every vertex Maker has claimed in $D$, the probability that it is a neighbor of $v$ is at least $\delta^{2} / 2$. Thus the expected number of 
neighbors of $v$ in $D$ is at least $\delta^{2} / 2 \cdot|D|=\delta^{2} / 2 \cdot 100 \log _{2} n / \delta^{2}=50 \log _{2} n$. Thus a standard Chernoff bound argument implies (P1).

By (P1), the minimum degree of Maker's graph is $\Omega(\log n)$. On the other hand, Maker's graph is of order $O(\log n)$, since the whole game ends in $O(\log n)$ rounds. Therefore Maker's graph (even minus the special edge $u v$ ) is connected and is of linear minimum degree (with respect to the order of the graph), which implies that its diameter is constant (see e.g, [5] Problem 2.1.65). Therefore, removing the edge $u v$, taking a shortest path between $u, v$ and adding $u v$ back, we obtain an odd cycle of constant length.

Acknowledgments: A major part of this work was done when the authors participated in the 4th Emléktábla workshop at Lake Balaton. The authors wish to thank Balázs Patkós and Dömötör Pálvölgyi for organizing this very nice event.

\section{References}

[1] N. Alon, D. Hefetz and M. Krivelevich, Playing to retain the advantage. Combinatorics, Probability and Computing 19 (2010), 481-491.

[2] T. Bohman, A. Frieze, M. Krivelevich and R. Martin, Adding random edges to dense graphs. Random Structures and Algorithms 24 (2004), 105-117.

[3] R. Diestel, Graph Theory. Springer-Verlag, 4th edition, (2010).

[4] D. Duffus, T. Łuczak and V. Rödl, Biased positional games on hypergraphs, Studia Scientarum Matematicarum Hungarica 34 (1998), 141-149.

[5] D. B. West, Introduction to Graph Theory. Prentice Hall, 2001. 